\title{
A theory-based online health behaviour intervention for new university students (U@Uni): results from a randomised controlled trial
}

Tracy Epton ${ }^{1,2^{*}}$, Paul Norman ${ }^{1}$, Aba-Sah Dadzie ${ }^{3}$, Peter R Harris ${ }^{4}$, Thomas L Webb ${ }^{1}$, Paschal Sheeran ${ }^{5}$, Steven A Julious ${ }^{6}$, Fabio Ciravegna ${ }^{3}$, Alan Brennan ${ }^{6}$, Petra S Meier $^{6}$, Declan Naughton ${ }^{7}$, Andrea Petroczi ${ }^{7}$, Jen Kruger ${ }^{6}$ and Iltaf Shah ${ }^{7}$

\begin{abstract}
Background: Too few young people engage in behaviours that reduce the risk of morbidity and premature mortality, such as eating healthily, being physically active, drinking sensibly and not smoking. This study sought to assess the efficacy and cost-effectiveness of a theory-based online health behaviour intervention (based on self-affirmation theory, the Theory of Planned Behaviour and implementation intentions) targeting these behaviours in new university students, in comparison to a measurement-only control.

Methods: Two-weeks before starting university all incoming undergraduates at the University of Sheffield were invited to take part in a study of new students' health behaviour. A randomised controlled design, with a baseline questionnaire, and two follow-ups ( 1 and 6 months after starting university), was used to evaluate the intervention. Primary outcomes were measures of the four health behaviours targeted by the intervention at 6-month follow-up, i.e., portions of fruit and vegetables, metabolic equivalent of tasks (physical activity), units of alcohol, and smoking status.
\end{abstract}

Results: The study recruited 1,445 students (intervention $n=736$, control $n=709,58 \%$ female, Mean age $=18.9$ years), of whom 1,107 completed at least one follow-up (23\% attrition). The intervention had a statistically significant effect on one primary outcome, smoking status at 6-month follow-up, with fewer smokers in the intervention arm (8.7\%) than in the control arm (13.0\%; Odds ratio $=1.92, p=.010)$. There were no significant intervention effects on the other primary outcomes (physical activity, alcohol or fruit and vegetable consumption) at 6-month follow-up.

Conclusions: The results of the RCT indicate that the online health behaviour intervention reduced smoking rates, but it had little effect on fruit and vegetable intake, physical activity or alcohol consumption, during the first six months at university. However, engagement with the intervention was low. Further research is needed before strong conclusions can be made regarding the likely effectiveness of the intervention to promote health lifestyle habits in new university students.

Trial registration: Current Controlled Trials, ISRCTN67684181.

Keywords: Young adults, Internet, Self-affirmation, Theory of planned behaviour, Implementation intentions, Alcohol, Fruit and vegetables, Exercise, Smoking

\footnotetext{
* Correspondence: epton.tracy@gmail.com

'Department of Psychology, University of Sheffield, Western Bank, Sheffield

S10 2TP, UK

${ }^{2}$ School of Psychological Sciences, University of Manchester, Oxford Road,

Manchester M13 9PL, UK

Full list of author information is available at the end of the article
} 


\section{Background}

Young people are at risk of developing serious health problems and diseases, such as cancer, heart and circulatory disease, obesity and type 2 diabetes, in the future due to their current lifestyle choices [1]. A recent health survey found that few young people in the UK perform recommended health behaviours that reduce these health risks, with only $20 \%$ of $16-24$ year olds reporting that they eat five portions of fruit and vegetables per day, less than $50 \%$ reporting that they meet weekly physical activity guidelines, $40 \%$ reporting that they exceed daily recommended alcohol limits, and 25\% reporting that they smoke tobacco [2]. Given the high percentages in some of these health risks, it is likely that many young people simultaneously engage in a variety of health-compromising behaviours. There is therefore a need for multi-behaviour health interventions aimed at young people. There is some evidence that multi-behaviour health interventions can have positive effects on lifestyle habits [3]. For example, successful multi-behaviour health interventions to promote both exercise and healthy diets have been reported for school children [4] and undergraduates [5]. This paper reports the results of a randomised controlled trial (RCT) to test the efficacy of a theory-based online intervention to promote healthy lifestyle habits (U@Uni) delivered during the transition from school to university [6]. To the best our knowledge, this is first test of a multi-behaviour health intervention delivered during this transition.

The transition from school to university may represent an ideal opportunity to deliver such interventions. First, it is possible to target a large proportion of young people in the UK. More than 350,000 students aged 20 or under start university each year, representing approximately $40 \%$ of school leavers [7]. Second, major life transitions, such as the move to university, represent a critical or "teachable" moment to intervene in order to promote healthy lifestyle habits. The inherent change in young peoples' environmental context, including the disruption of established peer networks, means that health beliefs and behaviours are likely to be in a state of flux and therefore more amenable to change [8,9]. Moreover, moving to a new location has been found to promote changes in behaviours such as quitting smoking [10].

Evidence indicates that the use of theory in the design of health behaviour interventions increases their efficacy [3]. The current intervention therefore included three theory-based techniques to promote health behaviour change. First, a self-affirmation manipulation was included to reduce defensive processing of health messages [11]. Second, theory-based messages were designed to increase motivation to adopt healthy behaviours [12]. Third, participants were prompted to form implementation intentions to help them to translate their intentions to change into behaviour [13].
The intervention was delivered online via a website and mobile app. The use of digital technologies to deliver a health behaviour intervention has a number of advantages [3]. In particular, the use of digital technologies means that it is possible to (a) deliver interventions to large numbers of people at relatively low cost, (b) ensure that the intervention is accessible 24 hours a day, so is available at critical moments, and (c) increase engagement through the use of interactive methods such as video streaming and sharing resources. In addition, the use of digital technologies may be particularly relevant to young people, who are the prime users of such technology $[14,15]$. A recent meta-analysis confirmed the potential of online health behaviour interventions, reporting a small but significant effect size $(d=0.16)$ on health behaviour [3].

The efficacy of the U@Uni health behaviour intervention, delivered shortly before students started university, was assessed using a randomised controlled design with follow-up 1 and 6-months after starting university. The two arms of the RCT were (i) an online intervention targeting four health behaviours and (ii) a measurement only control, with approximately $50 \%$ of participants randomly allocated to each condition. Full details of the intervention are provided in an earlier paper reporting the study protocol [6].

\section{Method \\ Participants and procedure}

Two weeks before starting university (September 2012), incoming undergraduate students to the University of Sheffield (all of whom were eligible to participate) $(N=4,611)$ were sent an email inviting them to take part in the U@Uni study, with a link to an online questionnaire with baseline measures of demographics, beliefs, and behaviour. $N=1,445$ incoming students (Mean age = 18.9 years; $58 \%$ female) completed the baseline questionnaire and were randomly allocated to the intervention $(n=736)$ and control arms $(n=709)$ using the random function on SurveyGizmo [16]. As detailed in the study protocol [6], we assumed a $50 \%$ response rate to the initial email invite and $40 \%$ attrition at 6 -month follow-up. With an anticipated 4,000 eligible participants, this would result in a final sample of 1,200 for the proposed analyses. It was calculated that the trial would have at least $80 \%$ power to detect a small effect size $(d=0.20)$ at a two-tailed significance level of .0127 (adjusted for multiple primary outcomes). Informed consent was obtained on the first page of the questionnaire (participants indicated their consent to participate by clicking a button before they were permitted to proceed to the questionnaire). See Figure 1 for the flow of participants through the trial and Table 1 for details of the baseline sample. 


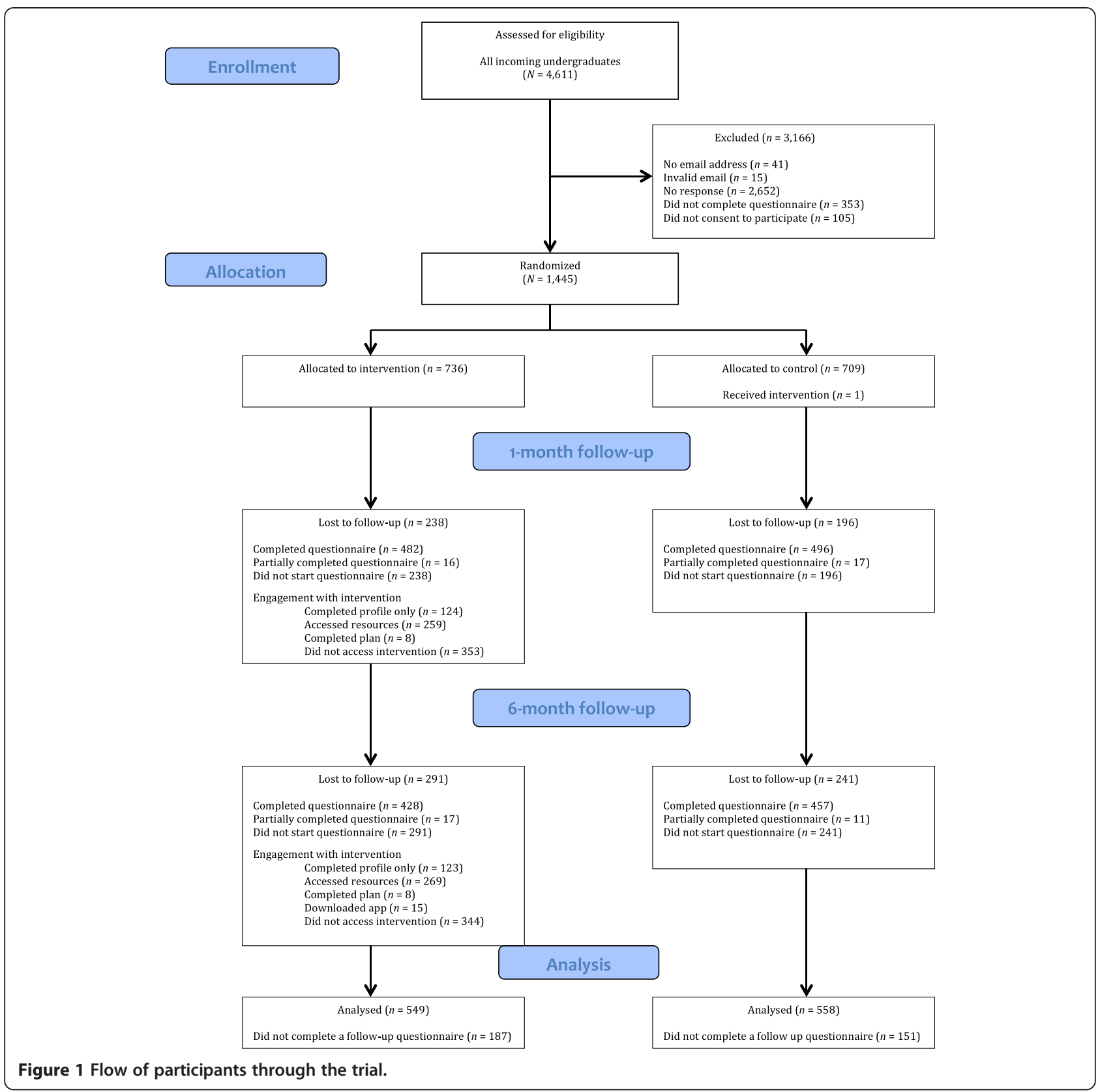

Participants assigned to the intervention arm were directed to the U@Uni website and asked to complete a profile page that contained the self-affirmation manipulation. After completing their profile, participants were asked to sign in to the website and view the online resources, which included theory-based messages (i.e., text, videos and links to further information) relevant to each of the four targeted health behaviours and a planner that contained instructions to form implementation intentions. Participants were able to selectively access information that was of interest to them and also had the opportunity to access more detailed information (using links to further information or via a search function).
Intervention participants were emailed prior to the start of the second university semester and invited to download a smartphone app designed for the Android operating system from the U@Uni website. The app and the website were accessible to intervention participants throughout the academic year.

All participants were asked to complete a follow-up questionnaire 1-month (October 2012) and 6-months (March 2013) after starting university. Participants were paid $£ 10$ for completing all three questionnaires and were entered into a $£ 100$ prize draw for each questionnaire they completed.

Participants were also sent emails when they started university and 6-months later inviting them to participate 
Table 1 Baseline characteristics of the sample

\begin{tabular}{|c|c|c|c|c|c|c|}
\hline \multirow[t]{2}{*}{ Variable } & \multicolumn{3}{|c|}{ Control } & \multicolumn{3}{|c|}{ Intervention } \\
\hline & $\%$ or mean & $S D$ & $N$ & $\%$ or mean & $S D$ & $N$ \\
\hline \multicolumn{7}{|l|}{ Demographic } \\
\hline \multicolumn{7}{|l|}{ Nationality } \\
\hline UK & 75.74 & - & 537 & 74.46 & - & 548 \\
\hline Non UK & 24.26 & - & 172 & 25.54 & - & 188 \\
\hline \multicolumn{7}{|l|}{ Ethnicity } \\
\hline White British & 67.42 & - & 476 & 65.98 & - & 483 \\
\hline White other & 5.95 & - & 42 & 6.97 & - & 51 \\
\hline Mixed & 3.97 & - & 28 & 2.46 & - & 18 \\
\hline Asian and Asian British & 8.64 & - & 61 & 8.61 & - & 63 \\
\hline Black and Black British & 2.27 & - & 16 & 2.46 & - & 18 \\
\hline Chinese & 10.48 & - & 74 & 12.16 & - & 89 \\
\hline Other & 1.27 & - & 9 & 1.37 & - & 10 \\
\hline \multicolumn{7}{|l|}{ Gender } \\
\hline Female & 55.15 & - & 391 & 61.55 & - & 453 \\
\hline Male & 44.85 & - & 318 & 38.45 & - & 283 \\
\hline Age & 19.04 & 2.91 & 709 & 18.76 & 1.99 & 736 \\
\hline \multicolumn{7}{|l|}{ Fruit and vegetable intake } \\
\hline Mean portions & 6.36 & 4.96 & 669 & 6.67 & 5.17 & 701 \\
\hline \multicolumn{7}{|l|}{ Physical activity } \\
\hline METS & 3402.37 & 5101.72 & 688 & 3140.11 & 3861.42 & 709 \\
\hline Mean hours sitting & 344.36 & 179.09 & 609 & 336.17 & 171.42 & 641 \\
\hline \multicolumn{7}{|l|}{ Alcohol consumption } \\
\hline Mean total units in last 7 days & 11.88 & 18.54 & 708 & 11.17 & 18.72 & 736 \\
\hline Mean number of days binge drinking in last 7 days (drinkers only) & 1.00 & 1.04 & 447 & 1.04 & 1.14 & 425 \\
\hline Mean alcohol objective (FAEE) & 2.07 & 2.02 & 54 & 2.08 & 2.56 & 54 \\
\hline \multicolumn{7}{|l|}{ Smoking } \\
\hline Has smoked & 37.24 & - & 264 & 37.23 & - & 274 \\
\hline Has never smoked & 62.76 & - & 445 & 62.77 & - & 462 \\
\hline Current smokers & 11.99 & - & 85 & 11.28 & - & 83 \\
\hline Not a current smoker & 88.01 & - & 624 & 88.72 & - & 653 \\
\hline Cigarettes smoked per week & 3.19 & 14.12 & 496 & 1.88 & 10.95 & 483 \\
\hline Smoking objective (cotinine) & .48 & .49 & 54 & .45 & .42 & 54 \\
\hline Smoking objective (nicotine) & 7.19 & 18.67 & 54 & 9.49 & 19.16 & 54 \\
\hline \multicolumn{7}{|l|}{ Other outcomes } \\
\hline \multicolumn{7}{|l|}{$E Q-5 D-3 L$} \\
\hline Mean health index scores from EQ-5D-3L (VAS) & .90 & .14 & 708 & .91 & .14 & 735 \\
\hline Mean health index score from EQ-5D-3L (TTO) & .92 & .13 & 708 & .92 & .14 & 735 \\
\hline Mean EQ-5D-3L visual analogue scale & 78.21 & 13.61 & 706 & 78.11 & 15.85 & 730 \\
\hline \multicolumn{7}{|l|}{ Recreational drugs } \\
\hline Have taken recreational drugs (SSC measure) ${ }^{1}$ & 18.73 & \pm 9.54 & 132 & 16.49 & \pm 9.56 & 121 \\
\hline Have not taken recreational drugs (SSC measure) & 81.27 & & 575 & 83.51 & & 613 \\
\hline Have taken recreational drugs (biochemical measure) & 39.53 & - & 17 & 45.71 & - & 16 \\
\hline Have not taken recreational drugs (biochemical measure) & 60.47 & - & 26 & 54.29 & - & 19 \\
\hline
\end{tabular}


Table 1 Baseline characteristics of the sample (Continued)

\begin{tabular}{|c|c|c|c|c|c|c|}
\hline \multicolumn{7}{|l|}{$B M I$} \\
\hline Mean BMI self-report & 22.06 & 3.71 & 662 & 22.24 & 3.81 & 695 \\
\hline Mean BMl objective & 22.28 & 3.85 & 54 & 22.06 & 3.88 & 54 \\
\hline \multicolumn{7}{|c|}{ Social cognition variables } \\
\hline \multicolumn{7}{|l|}{ Fruit \& veg } \\
\hline Self-efficacy & 5.71 & 1.48 & 703 & 5.80 & 1.36 & 733 \\
\hline Perceived control & 5.51 & 1.47 & 703 & 5.53 & 1.45 & 733 \\
\hline Intention & 5.24 & 1.47 & 703 & 5.39 & 1.45 & 733 \\
\hline \multicolumn{7}{|l|}{ Physical activity } \\
\hline Self-efficacy & 5.97 & 1.37 & 707 & 6.03 & 1.31 & 734 \\
\hline Perceived control & 5.66 & 1.46 & 707 & 5.58 & 1.46 & 734 \\
\hline Intention & 5.87 & 1.36 & 707 & 5.86 & 1.37 & 734 \\
\hline \multicolumn{7}{|l|}{ Binge drinking } \\
\hline Self-efficacy & 5.48 & 2.15 & 705 & 5.38 & 2.20 & 732 \\
\hline Perceived control & 5.94 & 1.55 & 705 & 6.05 & 1.43 & 732 \\
\hline Intention & 3.20 & 1.96 & 705 & 3.12 & 1.99 & 732 \\
\hline \multicolumn{7}{|l|}{ Smoking } \\
\hline Self-efficacy & 4.94 & 2.48 & 706 & 4.90 & 2.51 & 733 \\
\hline Perceived control & 6.39 & 1.45 & 706 & 6.51 & 1.27 & 733 \\
\hline Intention & 1.56 & 1.41 & 706 & 1.52 & 1.30 & 733 \\
\hline
\end{tabular}

${ }^{1}$ Includes confidence interval. Notes. METS = metabolic equivalent of task, FAEE = fatty acid ethyl esters, VAS = visual analogue scale technique, TTO = time trade-off technique, $\mathrm{SSC}=$ single count method, $\mathrm{BMI}=$ body mass index.

in an additional study on the biochemical markers of health behaviour. A sample of 108 students (Intervention $n=54$, Control $n=54$, Mean age $=19.58$ years, $S D=3.83$ ) was recruited to this additional study at baseline and 78 of these also provided a hair sample at 6-month follow-up (Intervention $n=35$, Control $n=43$ ).

Ethical approval for the study was obtained from the Department of Psychology Research Ethics Committee at the University of Sheffield.

\section{Intervention materials}

Full details of the intervention are provided in the protocol paper [6].

\section{Self-affirmation task}

The self-affirmation manipulation was adapted from an existing value-affirmation task [17] and embedded in a profile page. Participants were presented with a list of eight commonly held personal values (sense of humour, academic achievement, relations with family and friends, social skills, spontaneity, artistic skills/aesthetic appreciation, religion/faith/spirituality, and respect/decency/manners) and asked to select their most important value (or provide their own) and to briefly explain why the value was important to them.

\section{Theory-based messages}

Theory-based persuasive messages were developed to encourage regular exercise and fruit and vegetable intake, and to discourage binge drinking and smoking. The messages were based on the Theory of Planned Behaviour [18] and developed on the basis of formative work that identified the key behavioural, normative and control beliefs associated with intentions to perform each of the four health behaviours in new university students [see 19, for details of the health message development process]. The messages included a mixture of text and videos, as well as links to other relevant material.

\section{Implementation intentions}

The planner comprised a series of drop down menus that helped participants to form implementation intentions by asking them to identify (i) a good opportunity to act on their intentions (e.g., when tempted to binge drink) and (ii) a suitable response to their identified opportunity (e.g., to remind themselves that they have lectures tomorrow) for each of the four targeted health behaviours. The planner also allowed participants to type in their own opportunities and responses. The plans were stored in a 'plan repository' and participants were also able to opt to have a reminder of each plan emailed to them. 


\section{Measures}

The four primary outcome measures were (i) portions of fruit and vegetables per day, (ii) physical activity in the last week, (iii) alcohol consumption in the last week, and (iv) smoking status at 6-month follow-up. A range of secondary outcome measures was also assessed as detailed below. Unless indicated, all of the measures were taken at baseline as well as at 1 and 6-month follow-up.

\section{Fruit and vegetable intake}

Fruit and vegetable intake (portions per day) was measured with items based on the Health Survey for England (HSE) [2]. Participants were asked to think about the preceding day and indicate whether they had eaten any of nine different types of fruit and vegetables (e.g., "Did you eat any salad yesterday?") and if so how much of each type they had eaten (e.g., "How many cereal bowls of salad did you eat yesterday?").

\section{Physical activity}

The Short Form of International Physical Activity Questionnaire (IPAQ-SF) was used to assess levels of physical activity [20]. Respondents were asked to indicate how many times, and for how long, they had engaged in vigorous exercise (defined as "activities that take hard physical effort and make you breathe much harder than normal"), moderate exercise (defined as "activities that take moderate physical effort and make you breathe somewhat harder than normal") and walking in the past 7 days. Responses were converted into METs (metabolic equivalent of task) to provide a total IPAQ score. An additional question asked about sedentary activity. Data on use (membership and number of visits) of the university sports facilities were collected from automatic records kept by the university sports centre (users have to swipe a card when they attend).

\section{Alcohol}

Alcohol consumption was assessed using items from the General Lifestyle Survey [21] to provide a measure of units of alcohol per week and number of binge sessions per week (i.e., participants were asked to indicate on which days they had drunk alcohol in the last 7 days and the type and amount of alcohol that they drank on each day). The Alcohol Use Disorders Identification Test (AUDIT) [22] was used at the 6-month follow-up to assess hazardous and harmful patterns of alcohol use.

\section{Smoking}

Items based on the HSE were used to assess participants' current smoking status and the typical number of cigarettes/amount of tobacco that they smoked [2].

\section{Health status}

The EQ-5D-3L [23], a short standardized measure of health status, was used to assess levels of severity (no problems/some or moderate problems/extreme problems) in five dimensions: mobility, self-care, usual activities, pain/ discomfort, and anxiety/depression. The measure provides a descriptive profile and a single index value for health status and is recommended as the measure of healthrelated quality of life for health economic evaluations in the UK [24].

\section{Recreational drug use}

A Single Sample Count Method [25] was used to estimate the prevalence of recreational drug use in the sample. Respondents were asked to indicate the number of "yes" answers (0 or 5, 1, 2, 3, 4) to five questions - four of which have a $50 \%$ population prevalence (e.g., odd or even date of birth) and one of which was about their use of recreational drugs. In this way it was possible to estimate the prevalence of recreational drug use in the sample without being able to identify whether individual participants do or do not use recreational drugs, as 50\% of the sample would answer "yes" answers to each of the four questions. This Single Sample Count Method has been shown to encourage accurate reporting of behaviours that are illegal and could be regarded as socially undesirable [26].

\section{$B M I$}

Participants recorded their height and weight, from which their BMI was calculated.

\section{Health services usage}

Participants were asked to report their use of health services (e.g., GP visits, hospitalizations) at the 6-month follow-up.

\section{Academic performance}

Average exam marks and registration status (i.e., registered level one passed, registered level one failed, withdrawn, leave of absence) were used to assess academic performance at the end of the academic year.

\section{Social cognitive variables}

Measures of social cognitive variables for each behaviour were included. Each variable was measured with one item per behaviour, except attitude, which was measured with two items. Measures of intention (e.g., "I intend to engage in regular exercise at university"), self-efficacy (e.g., "If I wanted, I could easily engage in regular exercise at university"), and perceived control (e.g., "Whether or not I engage in regular exercise at university is under my control") were taken at all time points. Measures of attitude (e.g., "Engaging in regular exercise at university 
would be... good/bad"), subjective norms (e.g., "Most people who are important to me think I should/should not engage in regular exercise at university) and planning (e.g., "To what extent do you have a detailed plan about how to engage in regular exercise at university?") were taken at the 1 and 6 -month follow-ups.

\section{Engagement with the digital intervention}

Measures of engagement with the intervention, for both the website and the mobile app, were (i) completion of the self-affirmation task (i.e., profile page), (ii) whether or not participants accessed the theory-based messages and (iii) the number of implementation intentions that were formed.

\section{Biochemical measures}

Participants recruited to the additional study on the biochemical markers of health behaviour provided a hair sample (3 $\mathrm{cm}$ long) that was liquefied and analysed for biochemical markers of various health behaviours related to alcohol consumption, cigarette smoking, and recreational drug use. Following extraction procedures, markers of alcohol (fatty acid ethyl esters [FAEE]) and cigarettes (nicotine, cotinine) were quantified using liquid chromatography with tandem mass spectrometric detection (LCMS/MS). In addition, evidence for recreational drug use was detected by screening for commonly used drugs and their metabolites. These included: amphetamine, 3,4methylenedioxyamphetamine (MDA), 3,4-methylenedioxy$\mathrm{N}$-methylamphetamine (MDMA), ephedrine, mephedrone, tetrahydrocannabinol (THC), cocaine, heroin, lysergic acid diethylamide (LSD), phencyclidine (PCP) and ketamine. Morphine, codeine, hydromorphone and hydrocodone were treated separately owing to their potential medical use (i.e., as a pain reliever or cough suppressant). A 6430 triple quadruple mass spectrometer (made by Agilent Technologies UK) was employed with a dynamicmultiple reaction monitoring-liquid chromatography mass spectrometry (DYN-MRM-LC-MS/MS) method. Participants recruited to this additional study also had their height and weight measured to calculate an objective measure of BMI.

\section{Statistical analysis}

Analysis of the 6-month data was conducted using an intention-to-treat approach (i.e., data were included from all participants who completed at least one followup survey); missing data at 6-months were imputed from the 1-month follow-up data by carrying the last observation forward $[27,28]^{\mathrm{a}}$. Analysis of the 1-month data was based only on participants who completed the 1-month survey.

A series of analyses of covariance (ANCOVA) and logistic regression analyses were used to assess the impact of the intervention on performance of the targeted behaviours, controlling for corresponding baseline scores, gender, age and nationality (i.e., UK or non-UK). For primary outcomes, the bonferroni correction was used; thus statistical significance was declared if any of the primary endpoints were significant at .0127 to account for multiple tests.

The impact of the intervention on secondary outcomes (i.e., health behaviours at 1-month follow-up, social cognitive variables, health status, recreational drug use, BMI, health services usage, academic performance, use of university sports facilities and biochemical measures) was assessed using a similar analysis strategy, i.e., using ANCOVAs and logistic regression analyses that controlled for corresponding baseline scores (where available), gender, age and nationality. As these were secondary outcomes no adjustments were made for multiple tests. The analyses were repeated to (i) assess the effect of engagement with the intervention (per protocol analyses) and (ii) to assess the effect of moderators (with dichotomised moderators as additional IVs). Additional analyses were conducted to compare dropouts and completers on the baseline measures.

\section{Results}

\section{Randomisation check}

There were no differences between participants in the intervention and control arms in baseline measures of the four health behaviours. Gender and age did, however, differ between the two arms (see Table 1), with more females and younger participants in the intervention arm than in the control arm.

\section{Primary outcomes}

At 6-month follow-up, the intervention and control arms had statistically significant differences in the number of current smokers (B =.65, SE $=.25, p=.010) ; 8.70 \%$ of participants in the intervention arm reported that they were current smokers compared to $13.01 \%$ of participants in the control arm. More detailed analyses revealed that, of the 480 non-smokers in the intervention arm at baseline, 14 (2.92\%) were smokers at 6-month follow-up. In contrast, of the 489 non-smokers in the control arm at baseline, 27 (5.52\%) were smokers at 6-month follow-up. In addition, of the 60 smokers in the intervention arm at baseline, $27(45.00 \%)$ were non-smokers at 6-month follow-up. In contrast, of the 64 smokers in the control arm at baseline, 19 (29.69\%) were nonsmokers at 6-month follow-up.

Fruit and vegetable intake, physical activity, and alcohol consumption at 6-month follow-up did not differ significantly between the two arms (see Table 2). 
Table 2 Estimated marginal means, percentages, sample sizes, standard deviations, differences, p values and effect sizes for primary and secondary outcomes

\begin{tabular}{|c|c|c|c|c|c|c|c|c|c|c|c|c|c|c|c|c|c|c|}
\hline \multirow{3}{*}{ Variable } & \multicolumn{8}{|c|}{ 1-month follow-up } & \multicolumn{9}{|c|}{ 6-month follow-up } & \multirow[b]{3}{*}{$d$} \\
\hline & \multicolumn{3}{|c|}{ Control } & \multicolumn{3}{|c|}{ Intervention } & \multirow[b]{2}{*}{ Diff } & \multirow[b]{2}{*}{$p$} & \multirow[b]{2}{*}{$d$} & \multicolumn{3}{|c|}{ Control } & \multicolumn{3}{|c|}{ Intervention } & \multirow[b]{2}{*}{ Diff } & \multirow[b]{2}{*}{$p$} & \\
\hline & $\begin{array}{l}\% \text { or } \\
\text { Mean }\end{array}$ & $S D$ & $n$ & $\begin{array}{l}\% \text { or } \\
\text { Mean }\end{array}$ & $S D$ & $n$ & & & & $\begin{array}{l}\% \text { or } \\
\text { Mean }\end{array}$ & $S D$ & $n$ & $\begin{array}{l}\% \text { or } \\
\text { Mean }\end{array}$ & $S D$ & $n$ & & & \\
\hline \multicolumn{19}{|l|}{ Fruit and vegetable intake } \\
\hline Mean portions & 5.47 & 4.26 & 453 & 6.02 & 4.18 & 436 & .55 & .053 & 0.13 & 5.72 & 4.98 & 512 & 5.61 & 4.89 & 495 & -.11 & .708 & -0.02 \\
\hline \multicolumn{19}{|l|}{ Physical activity } \\
\hline Mean METS & 2563.39 & 2162.88 & 471 & 2755.63 & 2169.40 & 452 & 192.24 & .179 & 0.09 & 3316.10 & 5143.79 & 526 & 3350.52 & 5144.16 & 513 & 34.42 & .914 & 0.01 \\
\hline Mean hours sitting & 400.55 & 150.49 & 388 & 396.19 & 150.44 & 372 & -4.36 & .890 & 0.03 & 408.12 & 158.25 & 450 & 412.24 & 158.30 & 435 & 4.12 & .699 & -0.03 \\
\hline Member of Sport Sheffield & & & & & & & & & & 56.52 & - & 325 & 54.92 & - & 318 & -1.60 & .392 & -0.04 \\
\hline Not a member of Sport Sheffield & & & & & & & & & & 43.48 & - & 250 & 45.08 & - & 261 & & & OR 1.11 \\
\hline Mean Sport Sheffield attendance & & & & & & & & & & 8.15 & 15.50 & 325 & 9.92 & 15.34 & 318 & 1.77 & .149 & 0.15 \\
\hline \multicolumn{19}{|l|}{ Alcohol consumption } \\
\hline Mean units in last 7 days & 13.86 & 16.89 & 507 & 12.55 & 17.06 & 491 & .02 & .222 & 0.08 & 13.41 & 19.65 & 547 & 13.01 & 19.75 & 540 & -.40 & .737 & 0.02 \\
\hline $\begin{array}{l}\text { Mean number of days binge drinking } \\
\text { in last } 7 \text { days (drinkers only) }\end{array}$ & 1.33 & .99 & 271 & 1.31 & .92 & 234 & -.02 & .901 & 0.02 & 1.16 & .89 & 319 & 1.16 & .85 & 288 & .00 & .973 & 0.00 \\
\hline \multicolumn{19}{|l|}{ AUDIT } \\
\hline Mean consumption & & & & & & & & & & 6.45 & 2.60 & 400 & 6.32 & 2.52 & 376 & -.13 & .480 & 0.05 \\
\hline Mean dependency & & & & & & & & & & .93 & 1.39 & 393 & .88 & 1.34 & 366 & -.05 & .597 & 0.04 \\
\hline Mean problems & & & & & & & & & & 2.26 & 2.60 & 393 & 2.05 & 2.49 & 366 & -.21 & .256 & 0.08 \\
\hline Mean alcohol objective (FAEE) & & & & & & & & & & 2.55 & 4.59 & 43 & 2.80 & 4.61 & 34 & .25 & .816 & -0.05 \\
\hline \multicolumn{19}{|l|}{ Smoking } \\
\hline Has smoked & 42.37 & - & 211 & 40.41 & - & 196 & -1.96 & .849 & 0.04 & 47.74 & - & 264 & 46.30 & - & 250 & -1.44 & .776 & 0.03 \\
\hline Has never smoked & 57.63 & - & 287 & 59.59 & - & 289 & 1.96 & & OR 1.04 & 52.26 & - & 289 & 53.70 & - & 290 & 1.44 & & OR 1.05 \\
\hline Smoked since attending University & 25.96 & - & 129 & 24.33 & - & 118 & -1.63 & .578 & 0.05 & 35.51 & - & 196 & 35.93 & - & 194 & .42 & .878 & -0.01 \\
\hline Not smoked since starting University & 74.04 & - & 368 & 75.67 & - & 367 & 1.63 & & OR 1.09 & 64.49 & - & 356 & 64.07 & - & 346 & -.42 & & OR 0.98 \\
\hline Current smoker & 11.45 & - & 57 & 9.07 & - & 44 & -2.38 & .333 & 0.14 & 13.02 & - & 72 & 8.70 & - & 47 & -4.32 & .010 & 0.25 \\
\hline Not a current smoker & 88.55 & - & 441 & 90.93 & - & 441 & 2.38 & & OR 1.36 & 86.98 & - & 481 & 91.30 & - & 493 & 4.32 & & OR 1.92 \\
\hline Mean cigarettes smoked per week & 2.74 & 5.57 & 496 & 2.35 & 5.71 & 483 & -.39 & .286 & 0.07 & 3.24 & 6.81 & 552 & 2.70 & 6.73 & 538 & -.54 & .181 & 0.08 \\
\hline Mean smoking objective (cotinine) & & & & & & & & & & .37 & .33 & 43 & .51 & .35 & 34 & .14 & .081 & -0.41 \\
\hline Mean smoking objective (nicotine) & & & & & & & & & & 7.24 & 21.44 & 43 & 12.85 & 21.52 & 34 & 5.61 & .266 & -0.26 \\
\hline
\end{tabular}

\section{Other outcomes}

\section{EQ-5D-3L}

Mean health index scores from

EQ-5D-3L(VAS) 
Table 2 Estimated marginal means, percentages, sample sizes, standard deviations, differences, $p$ values and effect sizes for primary and secondary outcomes (Continued)

\begin{tabular}{|c|c|c|c|c|c|c|c|c|c|c|c|c|c|c|c|c|c|c|}
\hline Mean health index scores from EQ-5D-3L (TTO) & .92 & .22 & 495 & .92 & .22 & 482 & 0.00 & .728 & 0.00 & .92 & .23 & 542 & .92 & .23 & 530 & .00 & .739 & 0.00 \\
\hline Mean EQ-5D-3L visual analogue scale & 77.63 & 12.45 & 494 & 76.66 & 12.45 & 477 & -.97 & .224 & -0.08 & 77.69 & 13.01 & 540 & 77.17 & 13.05 & 524 & .01 & .508 & -0.04 \\
\hline \multicolumn{19}{|l|}{ Recreational drug use } \\
\hline Have taken recreational drugs (SSC measure) ${ }^{1}$ & 9.52 & \pm 10.74 & 47 & 22.57 & \pm 12.11 & 109 & 13.05 & $<.001$ & -0.57 & 8.27 & \pm 11.12 & 38 & 24.36 & \pm 12.7 & 105 & 16.09 & $<.001$ & -0.70 \\
\hline $\begin{array}{l}\text { Have not taken recreational drugs } \\
\text { (SSC measure) }\end{array}$ & 90.48 & & 450 & 77.43 & & 373 & -13.05 & & OR 2.04 & 91.73 & & 418 & 75.64 & & 324 & -16.09 & & OR 2.39 \\
\hline $\begin{array}{l}\text { Have taken recreational drugs (biochemical } \\
\text { measure) }\end{array}$ & & & & & & & & & & 41.86 & - & 18 & 45.71 & - & 16 & 3.85 & .491 & -0.09 \\
\hline $\begin{array}{l}\text { Have not taken recreational drugs } \\
\text { (biochemical measure) }\end{array}$ & & & & & & & & & & 58.14 & & 25 & 54.29 & - & 19 & -3.85 & & OR 1.29 \\
\hline \multicolumn{19}{|l|}{ BMI } \\
\hline Mean BMl & 21.97 & 2.96 & 448 & 22.22 & 3.15 & 440 & .25 & .230 & -0.08 & 22.15 & 2.23 & 499 & 22.12 & 2.22 & 494 & -.03 & .870 & 0.01 \\
\hline Mean objective BMI & & & & & & & & & & 22.01 & 2.90 & 100 & 22.27 & 2.86 & 91 & .01 & .550 & -0.09 \\
\hline \multicolumn{19}{|l|}{ Health service usage ${ }^{2}$} \\
\hline Mean times visited GP in last 6 months & & & & & & & & & & 1.33 & 1.49 & 455 & 1.18 & 1.66 & 429 & .01 & .169 & 0.10 \\
\hline Alcohol intervention offered by GP & & & & & & & & & & .39 & - & 1 & .82 & - & 2 & .43 & .648 & -0.41 \\
\hline Alcohol intervention not offered by GP & & & & & & & & & & 99.61 & - & 253 & 99.18 & - & 241 & -.43 & & OR 1.68 \\
\hline Attended alcohol intervention & & & & & & & & & & 0 & - & 0 & 0 & - & 0 & .00 & - & \\
\hline Did not attend alcohol intervention & & & & & & & & & & 100 & - & 1 & 100 & - & 2 & .00 & & \\
\hline Mean times visited A\&E & & & & & & & & & & .10 & .43 & 452 & .10 & .41 & 426 & .00 & .882 & 0.00 \\
\hline Mean times admitted to A\&E & & & & & & & & & & .19 & .52 & 42 & .25 & .51 & 40 & .06 & .622 & -0.12 \\
\hline Mean times required an ambulance & & & & & & & & & & .03 & .21 & 429 & .03 & .20 & 404 & .02 & .802 & 0.00 \\
\hline Mean times admitted to hospital & & & & & & & & & & .04 & .21 & 450 & .06 & .21 & 425 & .02 & .253 & -0.10 \\
\hline Mean elective admissions to hospital & & & & & & & & & & .34 & .69 & 13 & .68 & .67 & 20 & .34 & .181 & -0.50 \\
\hline Mean non-elective admissions to hospital & & & & & & & & & & 1.02 & .66 & 12 & .44 & .63 & 20 & -.58 & .020 & 0.90 \\
\hline $\begin{array}{l}\text { Mean other times visited hospital } \\
\text { (not incl. above) }\end{array}$ & & & & & & & & & & .25 & .85 & 447 & .22 & .82 & 424 & -.03 & .642 & 0.04 \\
\hline \multicolumn{19}{|l|}{ Academic achievement } \\
\hline Mean grade of academic year & & & & & & & & & & 62.49 & 8.99 & 481 & 62.35 & 9.13 & 473 & -.14 & .814 & -0.02 \\
\hline Progressed from level one & & & & & & & & & & 96.54 & & 446 & 95.45 & & 435 & -.09 & .810 & -0.01 \\
\hline Did not progress & & & & & & & & & & 3.46 & - & 16 & 3.55 & & 16 & .09 & & OR 1.01 \\
\hline
\end{tabular}

Social cognition variables

Fruit and vegetables

Mean descriptive norm 
Table 2 Estimated marginal means, percentages, sample sizes, standard deviations, differences, $p$ values and effect sizes for primary and secondary outcomes (Continued)

\begin{tabular}{|c|c|c|c|c|c|c|c|c|c|c|c|c|c|c|c|c|c|c|}
\hline Mean injunctive norm & 6.05 & 1.10 & 488 & 5.93 & 1.08 & 478 & -.12 & .116 & -0.11 & 3.18 & 1.62 & 534 & 3.43 & 1.61 & 527 & .25 & .017 & 0.15 \\
\hline Mean perceived control & 5.63 & 1.33 & 488 & 5.65 & 1.31 & 478 & .02 & .785 & 0.02 & 5.66 & 1.39 & 534 & 5.85 & 1.38 & 527 & .19 & .018 & 0.14 \\
\hline Mean self-efficacy & 5.50 & 1.55 & 488 & 5.49 & 1.53 & 478 & -.01 & .944 & -0.01 & 5.58 & 1.39 & 534 & 5.72 & 1.38 & 527 & .14 & .106 & 0.10 \\
\hline Mean intention & 5.39 & 1.10 & 488 & 5.31 & 1.09 & 478 & -.08 & .270 & -0.07 & 5.52 & 1.16 & 534 & 5.47 & 1.15 & 527 & -.05 & .442 & -0.04 \\
\hline Mean plan & 4.85 & 1.55 & 488 & 4.78 & 1.53 & 478 & -.07 & .474 & -0.05 & 5.09 & 1.39 & 534 & 5.13 & 1.38 & 527 & .04 & .628 & 0.03 \\
\hline Mean attitude & 6.22 & .88 & 488 & 6.20 & .87 & 478 & -.22 & .652 & -0.02 & 6.65 & 69 & 534 & 6.59 & .69 & 527 & -.06 & .208 & -0.09 \\
\hline \multicolumn{19}{|l|}{ Physical activity } \\
\hline Mean descriptive norm & 3.75 & 1.33 & 493 & 3.78 & 1.31 & 480 & .03 & .637 & 0.02 & 5.71 & 1.39 & 539 & 5.65 & 1.38 & 527 & -.06 & .530 & -0.04 \\
\hline Mean injunctive norm & 6.04 & 1.11 & 493 & 5.99 & 1.31 & 480 & -.05 & .550 & -0.04 & 4.10 & 1.63 & 539 & 4.20 & 1.61 & 527 & .10 & .288 & 0.06 \\
\hline Mean perceived control & 5.80 & 1.11 & 493 & 5.93 & 1.10 & 480 & .13 & .078 & 0.12 & 5.83 & 1.16 & 539 & 5.96 & 1.15 & 527 & .13 & .069 & 0.11 \\
\hline Mean self-efficacy & 5.62 & 1.33 & 493 & 5.82 & 1.31 & 480 & .20 & .020 & 0.15 & 5.78 & 1.39 & 539 & 5.83 & 1.38 & 527 & .05 & .523 & 0.04 \\
\hline Mean intention & 5.63 & 1.11 & 493 & 5.64 & 1.10 & 480 & .01 & .921 & 0.01 & 5.67 & 1.16 & 539 & 5.74 & 1.15 & 527 & .07 & .306 & 0.06 \\
\hline Mean plan & 4.94 & 1.55 & 493 & 5.04 & 1.53 & 480 & .08 & .338 & 0.06 & 5.14 & 1.63 & 539 & 5.13 & 1.61 & 527 & -.01 & .944 & -0.01 \\
\hline Mean attitude & 6.10 & .89 & 493 & 6.18 & .88 & 480 & .08 & .119 & 0.09 & 6.63 & .70 & 539 & 6.62 & .69 & 527 & .01 & .948 & -0.01 \\
\hline \multicolumn{19}{|l|}{ Binge drinking } \\
\hline Mean descriptive norm & 5.70 & 1.11 & 491 & 5.59 & 1.31 & 479 & -.11 & .177 & 0.09 & 2.79 & 1.85 & 537 & 3.00 & 1.83 & 525 & .21 & .059 & -0.06 \\
\hline Mean injunctive norm & 2.48 & 1.33 & 491 & 2.47 & 1.31 & 479 & -.01 & .974 & 0.01 & 5.10 & 1.62 & 537 & 4.93 & 1.83 & 525 & -.17 & .110 & 0.10 \\
\hline Mean perceived control & 6.24 & 1.11 & 491 & 6.13 & 1.09 & 479 & -.11 & .171 & -0.01 & 6.27 & 1.16 & 537 & 6.14 & 1.15 & 525 & -.13 & .097 & -0.10 \\
\hline Mean self-efficacy & 5.70 & 1.55 & 491 & 5.66 & 1.53 & 479 & -.04 & .704 & -0.03 & 5.81 & 1.39 & 537 & 5.69 & 1.38 & 525 & -.12 & .194 & -0.09 \\
\hline Mean intention & 3.18 & 1.11 & 491 & 3.16 & 1.31 & 479 & -.02 & .824 & 0.02 & 3.13 & 1.39 & 537 & 3.07 & 1.37 & 525 & -.06 & .457 & 0.04 \\
\hline Mean plan & 4.66 & 1.99 & 491 & 4.45 & 1.97 & 479 & -.21 & .115 & -0.11 & 4.89 & 2.09 & 537 & 4.50 & 2.06 & 525 & .39 & .003 & -0.19 \\
\hline Mean attitude & 2.50 & .89 & 491 & 2.52 & .88 & 479 & .02 & .819 & -0.02 & 1.83 & .93 & 537 & 1.91 & 1.15 & 525 & .09 & .207 & -0.08 \\
\hline \multicolumn{19}{|l|}{ Smoking } \\
\hline Mean descriptive norm & 4.19 & 1.33 & 488 & 4.05 & 1.31 & 479 & .14 & .113 & 0.11 & 1.73 & 1.39 & 537 & 1.87 & 1.37 & 525 & .14 & .105 & -0.10 \\
\hline Mean injunctive norm & 1.34 & .88 & 488 & 1.35 & .88 & 479 & .01 & .751 & -0.01 & 3.63 & 1.62 & 537 & 3.57 & 1.60 & 525 & -.06 & .593 & 0.04 \\
\hline Mean perceived control & 6.61 & 1.10 & 488 & 6.58 & 1.09 & 479 & .03 & .666 & -0.03 & 6.55 & 1.16 & 537 & 6.51 & 1.15 & 525 & -.04 & .518 & -0.03 \\
\hline Mean self-efficacy & 5.36 & 1.77 & 488 & 5.28 & 1.75 & 479 & -.08 & .490 & -0.05 & 5.12 & 1.62 & 537 & 5.37 & 1.83 & 525 & .25 & .172 & 0.14 \\
\hline Mean intention & 1.53 & .88 & 488 & 1.51 & .88 & 479 & -.02 & .671 & 0.02 & 1.61 & .93 & 537 & 1.53 & .92 & 525 & -.08 & .164 & 0.09 \\
\hline Mean plan & 5.58 & 2.21 & 488 & 5.30 & 2.19 & 479 & -.28 & .050 & -0.13 & 5.57 & 2.32 & 537 & 5.19 & 2.29 & 525 & -.38 & .003 & -0.16 \\
\hline Mean attitude & 1.45 & .66 & 488 & 1.47 & .66 & 479 & .02 & .573 & -0.03 & 1.32 & .70 & 537 & 1.35 & .69 & 525 & .03 & .491 & -0.04 \\
\hline
\end{tabular}

IIncludes confidence interval.

${ }^{2}$ These do not include imputed data as only measured at 6 months.

Notes. METS = metabolic equivalent of task, AUDIT = alcohol use disorder identification test FAEE = fatty acid ethyl esters, VAS = visual analogue scale technique, TTO = time trade-off technique SSC = single sample count, $\mathrm{OR}=$ odds ratio, $\mathrm{BMI}=$ body mass index, $\mathrm{GP}=$ general practitioner, $\mathrm{A \& E}=$ accident and emergency 


\section{Secondary outcomes}

The intervention and control arms had statistically significant differences in levels of recreational drug use at 1-month and 6-month follow-up. Using estimations from the Single Sample Count Method, there were more recreational drug users in the intervention than the control arm at both time points (see Table 2). Similar (but not statistically significant) differences observed between arms in biochemical markers of recreational drug use at 1-month and 6-month follow-up.

At 6-month follow-up there was a statistically significant difference between the intervention and control arms in the number of non-elective hospital admissions (see Table 2), with fewer admissions in the intervention arm. There were no differences in other measures of health services usage.

At 1 and/or 6-month follow-up, participants in the intervention and control arms did not significantly differ on secondary measures of physical activity (i.e., sedentary activity, walking activity, university sports centre membership and usage), alcohol use (i.e., number of binge drinking days, AUDIT or biochemical markers of alcohol), smoking (i.e., number of cigarettes smoked by smokers, biochemical markers of smoking), health status, BMI (self-report and objective), and academic achievement (i.e., status and grades).

There were some significant differences between the arms on the social cognitive variables at 6-month followup. In particular, participants in the intervention arm reported stronger injunctive norms and perceptions of control, but weaker descriptive norms, than participants in the control arm for fruit and vegetable intake. Participants in the intervention arm were less likely than participants in the control arm to report that they had a clear plan for avoiding smoking and binge drinking.

\section{Moderation and mediation analyses}

Deprivation index, gender, nationality (UK vs. non-UK), and ethnicity (white vs. non white) did not moderate the effect of the intervention on the primary outcome variables. Mediation analyses were not conducted as the intervention and control arm only differed for smoking status and participants in the intervention arm were less likely to report having a clear plan to avoid smoking (contrary to hypothesis).

\section{Engagement with the intervention}

There was low engagement with the intervention. At 6-month follow-up only 383 of the 736 participants allocated to the intervention arm (52\%) had completed the self-affirmation task, only 259 (35\%) had accessed the health messages, only 8 participants (1\%) had made a plan and only 15 participants $(2 \%)$ downloaded the app.

\section{Per protocol analysis}

To assess the effect of engagement with the intervention three per protocol analyses were conducted that included all participants in the control arm $(n=558)$ and (i) only those participants in the intervention arm who had completed the self-affirmation profile $(n=383)$ and (ii) only those participants in the intervention arm who had completed the self-affirmation profile and accessed the health messages $(n=259)$. There were no changes in the effects of the intervention on primary outcome variables when these per protocol analyses were conducted.

\section{Comparison of dropouts versus completers}

The 1,107 participants who completed at least one followup questionnaire differed from those who did not complete a follow-up questionnaire in nationality, $\chi^{2}(1, N=1445)=$ 19.91, $p<.001$, ethnicity, $\chi^{2}(1, N=1438)=30.76, p<.001$, gender, $\chi^{2}(1, N=1445)=45.37, p<.001$, baseline physical activity, $F(1,1397)=5.73, p=.017$, BMI, $F(1,1355)=4.01$, $p=.045$ and self-rated health status, $F(1,1434)=11.10$, $p=.001$ (see Additional file 1: Table S1). Completers were more likely to be British, white and female, do less exercise at baseline, have lower BMI and report that they were in better health, than those who did not complete a followup questionnaire. The differences in dropout rates between the two arms of the trial approached statistical significance, $\chi^{2}(1, N=1445)=3.40, p=.065$ (25.4\% intervention, $21.3 \%$ control).

\section{Discussion}

The U@Uni RCT assessed the efficacy of a theory-based online health behaviour intervention (based on self-affirmation theory, the Theory of Planned Behaviour and implementation intentions) compared to a measurement only control. The intervention targeted fruit and vegetable intake, physical activity, alcohol consumption, and smoking in new students and was delivered during the transition to university. The RCT included two follow-up assessments, at 1 and 6-months after starting university. The intervention reduced rates of smoking at the 6-month follow-up (8.70\% of participants in the intervention arm were smokers compared to $13.01 \%$ of the control arm) but did not affect fruit and vegetable intake, physical activity or alcohol consumption. These findings stand in contrast to the typical effects of online health behaviour interventions. For example, a meta-analysis by Webb et al. [3], found that Internetbased interventions tend to have small-sized effects on diet $(d=0.20)$, physical activity $(d=0.24)$, and alcohol consumption $(d=0.14)$, but a weaker effect on smoking behaviour $(d=0.07)$ than found in the current study $(d=0.25)$.

There are several possible reasons for the relatively weak effects found in the current trial. First, the U@Uni intervention targeted four health behaviours simultaneously. Webb et al. [3] found that interventions that targeted multiple health behaviours had smaller effects 
on health behaviour $(d=0.12)$ than those targeting a single health behaviour $(d=0.17)$. There are examples of successful multi-behaviour health interventions [4,5]; however, these focus solely on exercise and dietary behaviours that may be more complementary than those that also include health-risk behaviours such as alcohol consumption and smoking. Thus, the focus on multiple health behaviours may have diluted any intervention effects. This may have been amplified in the current intervention as participants had full control over the extent and type of information that they accessed on the intervention website. Second, there was low engagement with the intervention: only 383 (52\%) of the 736 participants allocated to the intervention arm completed the self-affirmation task, 259 (35\%) accessed the health messages, and $8(1 \%)$ made a plan. Indeed, analyses of the social cognitive variables indicated that participants in the intervention arm were less likely than participants in the control arm to report that they had a clear plan for avoiding smoking and binge drinking. Low engagement may have been due to three issues. First, the baseline questionnaire was time consuming, as extensive measures were taken for the four health behaviours and additional variables (e.g., health status, recreational drug use, social cognitions, demographics, etc.). As a result, participants may have been fatigued by the time that they were directed to the intervention website. Second, there were technical glitches with the intervention software that made it easy for participants to disengage from the intervention. For example, after completing the baseline questionnaire, participants in the intervention arm were redirected to the intervention website where they were asked to complete some login information before completing the self-affirmation task. After completing this task, participants had to login again to the intervention website to view the health messages. There was evidence that participants dropped out at each of these stages. Third, owing to technical delays, invitation emails were sent to potential participants only two weeks before the start of the university semester (i.e., one week before "Freshers' Week"). This likely coincided with a particularly busy time for many potential participants who may thus have failed to engage.

The secondary analyses revealed that, at least as estimated by the Single Sample Count Method [26], participants in the intervention arm were more likely to use recreational drugs than participants in the control arm at 1-month and 6-month follow-ups. Thus, there was some evidence that the intervention had undesired effects on non-targeted behaviours. Although, it is difficult to speculate how the intervention could lead to this undesired effect, one explanation for this effect is that participants in the intervention arm became more willing to take recreational drugs as they held a compensatory health belief that this unhealthy behaviour would be compensated for by their attempts to eat more fruit and vegetables, increase physical activity and reduce alcohol consumption [29]. No statistically significant differences were found between the two arms when biochemical markers of recreational drug use were considered, although the trend supported the results of the Single Count Method. However, given the low response rate to this aspect of the trial, this sample might be biased. The intervention had a mixed impact on the social cognitive variables. Participants in the intervention arm reported stronger injunctive norms and perceptions of control than participants in the control arm for fruit and vegetable intake. However, they also reported weaker descriptive norms for fruit and vegetable intake, and less clear plans for avoiding smoking and binge drinking. Finally, intervention participants reported fewer non-elective hospital admissions than the control arm at 6-month follow-up.

\section{Conclusions}

The initial findings reported in this paper suggest that the U@Uni intervention may be effective at reducing smoking, but has little effect on other targeted behaviours (fruit and vegetable intake, physical activity and alcohol consumption). However, this conclusion is tempered by a number of important limitations, most notably, low engagement with the intervention that, in part, was due to a number of minor technical glitches with the software platform used to deliver the intervention. The mixed results, coupled with low engagement, suggest that further research is needed before the efficacy (or lack of efficacy) of the U@Uni intervention can be confirmed. In particular, future research should (i) reduce the length of the baseline questionnaire, (ii) send out invitation emails earlier, so that potential participants have more time to engage with the intervention before starting university, and (iii) ensure that participants' experience of navigating through the intervention is optimised, so that they access more of the intervention material. A revised protocol for a repeat trial that incorporates $>$ these features is available from http://www.sheffield.ac.uk/polopoly_fs/ 1.373196!/file/UatUni2_Protocol.pdf.

\section{Endnotes}

${ }^{a}$ This follows the recommendations for clinical trials to only include participants who have completed one postintervention measure [28]. If baseline scores are used as a covariate in the analysis, replacing missing data with baseline data is problematic. This is due to the inflated correlation between the dependent variable and the baseline data that reduces the variance. This is particularly problematic with a large dropout rate such as that in this study of $23 \%$ [27]. 


\section{Additional file}

Additional file 1: Table S1. Baseline characteristics of the completers and dropouts table.

\section{Abbreviations}

ANCOVA: Analysis of covariance; AUDIT: Alcohol use disorder identification test; BMI: Body mass index; GLF: General lifestyle survey; HSE: Health survey for England; IPAQ-SF: International physical activity questionnaire-short form MANOVA: Multivariate analysis of variance; MET: Metabolic of equivalent task; QALY: Quality adjusted life years; RCT: Randomised controlled trial; TPB: Theory of Planned Behaviour.

\section{Competing interests}

The authors declare that they have no competing interests.

\section{Author's contributions}

TE was responsible for the data analysis, wrote the first draft of this paper and finalized the paper after feedback from all other authors. PN, PS, PH, $T L W, F C, A B, S J, D N, A P$, and PM designed and wrote the original proposal. $\mathrm{PN}, \mathrm{PS}, \mathrm{PH}, \mathrm{TLW}$ and TE were primarily responsible for the design of the intervention content, FC and ASD for the design of the data structure/model and build of the website and mobile app, PM, AB and JK for the health economics evaluation, SJ for the statistical analysis plan, AP for the indirect prevalence estimation of drug use, AP and DN for the biochemical markers aspect of the study and IS for the hair sample analyses. All authors commented on drafts and approved the final version of the paper.

\section{Acknowledgements}

The study was funded by the UK National Prevention Research Initiative (NPRI) Phase 4 (grant number: MR/J0004501/1). The NPRI includes the following Funding Partners (in alphabetical order): Alzheimer's Research Trust, Alzheimer's Society, Biotechnology and Biological Sciences Research Council, British Heart Foundation, Cancer Research UK, Chief Scientist Office, Scottish Government Health Directorate, Department of Health, Diabetes UK, Economic and Social Research Council, Health and Social Care Research and Development Division of the Public Health Agency (HSC \& R\&D Division), Medical Research Council, The Stroke Association, Wellcome Trust, Welsh Assembly Government and World Cancer Research Fund.

\section{Author details}

${ }^{1}$ Department of Psychology, University of Sheffield, Western Bank, Sheffield S10 2TP, UK. ${ }^{2}$ School of Psychological Sciences, University of Manchester, Oxford Road, Manchester M13 9PL, UK. ${ }^{3}$ Department of Computer Science, University of Sheffield, Regent Court, Sheffield S1 4DA, UK. ${ }^{4}$ School of Psychology, University of Sussex, Falmer BN1 9QH, UK. ${ }^{5}$ Psychology Department, University of North Carolina, 323 Davie Hall, Chapel Hill, NC 27599-3270, USA. '5Chool of Health and Related Research, University of Sheffield, Regent Court, Sheffield S1 4DA, UK. ${ }^{7}$ School of Life Science, Kingston University, Penrhyn Road, Kingston upon Thames KT1 2EE, UK.

Received: 31 January 2014 Accepted: 23 May 2014

Published: 5 June 2014

\section{References}

1. Parliamentary Office of Science and Technology: Health Behaviour. http:// www.parliament.uk/documents/post/postpn283.pdf.

2. National Centre for Social Research: Health survey for England. 2008. http:// www.ic.nhs.uk/statistics-and-data-collections/health-and-lifestyles-relatedsurveys/health-survey-for-england.

3. Webb TL, Joseph J, Yardley L, Michie S: Using the internet to promote health behavior change. J Med Internet Res 2010, 12:e4.

4. Jemmott JB III, Jemmott LS, O'Leary A, Ngwane A, Icard L, Bellamy S, Jones S, Landis JR, Heeren A, Tyler JC, Makwane MB: Cognitive-behavioura health-promotion intervention increases fruit and vegetable consumption and physical activity among South African Adolescents: a cluster-randomised controlled trial. Psych Health 2011, 26:167.

5. Zhang Y, Cooke R: Using a combined motivational and volitional intervention to promote exercise and healthy dietary behaviour among undergraduates. Diab Res Clin Pract 2012, 95:115.
6. Epton $T$, Norman $P$, Sheeran $P$, Harris PR, Webb TL, Ciravegna F, Meier $P$, Brennan A, Julious SA, Naughton D, Petroczi A, Dadzie AS, Kruger J: A theory based online health behaviour intervention for new university students: study protocol. BMC Publ Health 2013, 13:107.

7. UCAS: Final end of cycle figures for. 2012. http://www.ucas.com/data-analysis/ scheduled-releases\#endcycle.

8. Gill JS: Reported levels of alcohol consumption and binge drinking within the UK undergraduate student population over the last 25 years. Alcohol 2002, 37:109-120.

9. Wood W, Tam L, Witt MG: Changing circumstances, disrupting habits. J Pers Soc Psychol 2005, 88:918-933.

10. Heatherton TF, Nichols PA: Personal accounts of successful versus failed attempts at life change. Pers Soc Psychol Bull 1994, 20:664-675.

11. Harris PR, Epton T: The impact of self-affirmation on health cognition, health behavior and other health-related responses: a narrative review. Soc Pers Psychol Comp 2009, 3:962-978.

12. Conner M, Norman P: Predicting Health Behavior: Research and Practice with Social Cognition Models. 2nd edition. Buckingham: Open University Press; 2005.

13. Gollwitzer PM, Sheeran P: Implementation intentions and goal achievement: a meta-analysis of effects and processes. Adv Exp Soc Psychol 2006, 38:69-119.

14. Office for National Statistics: Internet access. http://www.ons.gov.uk/ons/rel/ rdit2/internet-access - households-and-individuals/2012/stb-internet-accesshouseholds-and-individuals-2012.html.

15. Ipsos Media CT: Technology tracker. http://www.ipsos-mori.com/ researchspecialisms/ipsosmediact/customresearch/technology/techtracker. aspx.

16. SurveyGizmo. http://www.surveygizmo.com.

17. Sherman DK, Bunyan DP, Creswell JD, Jaremka LM: Psychological vulnerability and stress: the effects of self-affirmation on sympathetic nervous system responses to naturalistic stressors. Health Psychol 2009 28:554-562.

18. Ajzen I: Attitudes, Personality and Behaviour. Milton Keynes, UK: Open University Press; 1988.

19. Epton $T$, Norman $P$, Harris $P R$, Webb $T L$, Snowsill FA, Sheeran $P$ : Development of theory-based messages: a three-phase programme of formative research. Health Promot Int. In press.

20. Booth ML: Assessment of physical activity: an international perspective. Res Q Exerc Sport 2000, 71:s114-s120.

21. Office for National Statistics: General Lifestyle Survey. 2010. http://www.ons. gov.uk/ons/rel/ghs/general-lifestyle-survey/2010/index.html.

22. Babor TF, Higgins-Biddle JC, Saunders JB, Monteiro MG: The alcohol use disorders identification test. whqlibdoc.who.int/hq/2001/who_msd_msb_01.6a.pdf.

23. Rabin R, de Charro F: EQ-5D: a measure of health status from the EuroQo Arm. Ann Med 2001, 23:337-343.

24. National Institute of Health and Clinical Excellence: Guide to the methods of technology appraisal. http://www.nice.org.uk/aboutnice/howwework/ devnicetech/guidetothemethodsoftechnologyappraisal.jsp.

25. Petroczi A, Nepusz T, Cross P, Taft H, Shah S, Deshmukh N, Schaffer J, Shane M, Adesanwo C, Barker J, Naughton DP: New non-randomised model to assess the prevalence of discriminating behaviour: a pilot study on mephedrone. Subst Abuse Treat Pr 2011, 3:6-20.

26. Nepusz T, Petróczi A, Naughton DP, Epton T, Norman P: Estimating the prevalence of socially sensitive behaviours: attributing guilty and innocent noncompliance with the single sample count method. Psychol Methods 2013. doi: 10.1037/a0034961.

27. Julious SJ, Mullen MA: Issues with using baseline in last observation carried forward analysis. Pharm Stat 2008, 7:146-146. doi:10.1002/pst.311.

28. International Conference on Harmonisation E9 Expert Working Group: ICH Harmonised tripartite guideline. Statistical principles for clinical trials. Stat Med 1999, 18:1905-1942

29. Rabia M, Knauper B, Miquelon P: The eternal quest for optimal balance between maximizing pleasure and minimizing harm: the compensatory health beliefs model. Brit J Health Psych 2006, 11:139-153.

doi:10.1186/1471-2458-14-563

Cite this article as: Epton et al:: A theory-based online health behaviour intervention for new university students (U@Uni): results from a randomised controlled trial. BMC Public Health 2014 14:563. 